\title{
Article \\ Performance Optimization of a Steam Generator Level Control System via a Revised Simplex Search-Based Data-Driven Optimization Methodology
}

\author{
Xiangsong Kong ${ }^{1,2, *}$, Changqing Shi ${ }^{1}$, Hang Liu ${ }^{1}$, Pengcheng Geng ${ }^{1,2}$, Jiabin Liu ${ }^{1}$ and Yasen Fan ${ }^{2}$ \\ 1 State Key Laboratory of Nuclear Power Safety Monitoring Technology and Equipment, China Nuclear Power \\ Engineering Co., Ltd., Shenzhen 518172, China; shichangqing@cgnpc.com.cn (C.S.); \\ liuhang@cgnpc.com.cn (H.L.); gengpengcheng2020@163.com (P.G.); liujiabin@cgnpc.com.cn (J.L.) \\ 2 School of Electrical Engineering and Automation, Xiamen University of Technology, Xiamen 361021, China; \\ 15259832896@163.com \\ * Correspondence: xskong@xmut.edu.cn
}

Citation: Kong, X.; Shi, C.; Liu, H.; Geng, P.; Liu, J.; Fan, Y. Performance Optimization of a Steam Generator Level Control System via a Revised Simplex Search-Based Data-Driven Optimization Methodology. Processes 2022, 10, 264. https://doi.org/ $10.3390 /$ pr10020264

Academic Editor: Zhiwei Gao

Received: 2 December 2021

Accepted: 21 January 2022

Published: 28 January 2022

Publisher's Note: MDPI stays neutral with regard to jurisdictional claims in published maps and institutional affiliations.

Copyright: (C) 2022 by the authors. Licensee MDPI, Basel, Switzerland. This article is an open access article distributed under the terms and conditions of the Creative Commons Attribution (CC BY) license (https:// creativecommons.org/licenses/by/ $4.0 /)$.

\begin{abstract}
A Steam generator is a crucial device of a nuclear power plant. Control performance of the steam generator level control system is key to its normal operation. To improve its performance, the control system parameters should be optimized by utilizing a proper optimization method. Furthermore, the method's efficiency is critical for its operability in the actual plant. However, the steam generator level process is a complex process, with high nonlinearity and time-varying properties. Traditional parameters tuning methods are experience-based, cumbersome, and time-consuming. To address the challenge, a systemic data-driven optimization methodology based on the modelfree optimization with a revised simplex search method was proposed. Rather than the traditional controller parameter tuning method, this method optimizes the control system directly by using control performance measurements. To strengthen its efficiency, two critical modifications were incorporated into the traditional simplex search method to form a knowledge-informed simplex search based on historical gradient approximations. Firstly, with the help of the historical gradient approximations, the revised method could sense the optimization direction more accurately and accomplish the iteration step size tuning adaptively, significantly reducing the optimization cost. Secondly, a revised iteration termination control strategy was developed and integrated to monitor the optimization progress, which can promptly terminate the progress to avoid unnecessary iteration costs. The effectiveness and the efficiency of the revised method were demonstrated through simulation experiments.
\end{abstract}

Keywords: performance optimization; steam generator level control; knowledge-informed simplex search method; historical iteration information; iteration termination control

\section{Introduction}

The steam generator (SG) is a critical component of the nuclear steam supply system in the nuclear island, which transfers heat from the primary loop to the secondary loop to produce steam [1]. Keeping the water level around preset programmed setpoints during the plant operation is of great importance. The water level requirement violation may jeopardize the plant's availability and safety. It was reported that about $25 \%$ of emergency shutdowns in the nuclear power plants were caused by poor control of the SG water level [2]. Hence, the performance of the steam generator level control is vital for plant safety, stability, and economical operation. Meanwhile, achieving the expected performance consists of a series of commissioning tests. The cost for a single test is high; thus, the efficiency of the performance optimization is of great concern. The number of the commissioning tests in optimization should be minimized. However, SG is a highly complex, nonlinear, time-varying system with inverse dynamics [3,4]. One of the dynamic features of the SG is 
the "shrink and swell" phenomenon. With this phenomenon, the water level may react temporarily in a reverse manner to water mass inventory changes. This complication may be accentuated during the plant start-up or low turbine load [5]. As a result, the performance optimization of the SG level control is challenging to accomplish.

For a nuclear power plant (NPP), the performance of the steam generator level control was greatly determined by several factors in different levels, including the process characteristics, the control system structure, and the controller parameters. To a particular steam generator, the process characteristics were relatively fixed. Once the structure of the control system is determined, the controller parameters are the only vital factors left. In this situation, the control system's performance usually has a close but underlying relationship with them. It is necessary to improve the performance by selecting the optimal settings efficiently. However, as their relationship is difficult to be obtained, searching for the optimal settings is usually experience-based, cumbersome, and time-consuming. There have been increasing demands to develop advanced methods to enhance the optimization efficiency.

Different kinds of methodologies used to search for the optimal controller settings can be summarized as below:

(1) Experience-based method

This type of method relies heavily on the engineers' experience. It does not rely on the controller performance model that it tries to search for the optimal settings directly in a model-free way. It can be called "Primitive model-free methods". These methods may include trial and error, the design of experiments (DOE), and the expert systembased control et al. They have the advantage of easy implementation. However, their disadvantages are apparent: they rely heavily on the experience of operators or experts, and they are usually cumbersome and time-consuming; moreover, they are challenging to obtain the real optimal settings, only suboptimal settings can be achieved.

(2) Model-based optimization

As a mainstream way for system optimization, model-based optimization relies on a performance model that relates the controller parameters and the control system performance. However, for the control parameters optimization, the relationship was too difficult to obtain, such that no accurate model could be built [6,7]. Nevertheless, the ProportionalIntegral-Derivative (PID) parameters tuning methods, such as the Ziegler-Nichols tuning method, could be treated as an approximate model-based method in essence [8-10]. For this method, the engineers should first obtain an accurate process model. Then a certain tuning formula can be developed based on the model. Finally, the parameters can be chosen according to the model coefficients and the formula. Hence, it can only provide relatively poor settings. The reason lies in the following: firstly, building an accurate model is often challenging; secondly, the formula is usually experience-dependent; thirdly, the parameter settings obtained are usually suboptimal [11].

(3) Intelligent optimization without model

With the development of artificial intelligence technology, intelligent optimization has gradually attracted more attention. For example, $\mathrm{Wu}$ et al. proposed to use a particle swarm optimization algorithm for the controller parameters optimization of an Advanced Passive pressurized water reactor-1000 (AP1000) plant [1]. The experimental results based on the AP1000 SG model indicated that the algorithm could achieve better control performance $[1,12]$. However, this algorithm needs a lot of batch experiments, which makes it impossible for the online optimization at the actual NPP plant.

(4) Data-driven control methods

As the industrial processes become more and more complex, data-driven control has emerged. The main approaches include iterative feedback tuning (IFT) $[13,14]$, virtual reference feedback tuning (VRFT) [15], and model-free adaptive control (MFAC) [16-18]. However, these methods mainly focus on the control strategy itself, and only covered limited parameters. As a complex process, in the actual NPP plant commissioning, there are various different kinds of parameters to be tuned, such as control logic parameters and 
time constants of the filters. Therefore, more general optimization methods in the upper level for system optimization that could cover more types of parameters are still needed.

All the above methods have their disadvantages or limitations, which lead to the inefficiency of the control performance optimization. A question emerged: is there a different way to promote the efficiency of the control performance optimization, considering that, by integrating the advantages of the above methods and avoiding their disadvantages, the efficiency may be promoted? Data-driven methods are powerful techniques for complex industrial automation processes [19]. Meanwhile, data-driven techniques have further stimulated research and applications of monitoring, optimization, and control. The data-driven method, integrating efficient optimization algorithms, becomes an attractive way [20-22] Hence, an analogous philosophy, integrating feasible data-based optimization algorithms without a prior model to formulate a data-driven optimization framework, is proposed to address the optimization of complex systems.

Kong had proposed a systematic and efficient simultaneous perturbation stochastic approximation (SPSA)-based optimization method for the steam generator level control and the injection molding quality control [23-26]. However, as a model-free method, the efficiency of the SPSA-based method still needs to be improved. Another selection was the simplex search-based model-free optimization (MFO), which usually behaves better than the SPSA at the low dimension circumstances. To improve the efficiency of the simplex search method (SS) on the medium-voltage insulator quality control, a revised simplex search method, knowledge-informed simplex search based on historical gradient approximations (GK-SS), has been proposed [27]. Similar to the idea of knowledge-based fault detection and diagnosis [28] and utilization of prior information [29], historical data generated during the data-driven optimization will be mined and utilized. In order to adapt to the characteristics of the control performance optimization problem and beef up the optimization efficiency, two critical modifications were formulated to the SS. Firstly, the historical-gradient approximations were utilized for the optimization search direction compensation. Secondly, an improved iteration termination control strategy, which uses the historical iteration information, was integrated to monitor the progress of the optimization. The two modifications are integrated to form a revised simplex search-based data-driven optimization methodology.

The remainder of this paper is organized as follows. Section 2 illustrates the performance optimization problem for the SG level control system. Section 3 presents an overview of the knowledge-informed optimization strategy, followed by the elementary procedure of the revised GK-SS method. The modifications on the search mechanism and the revised iteration termination control strategy are detailed. In Section 4, the simulation platform of the steam generator is demonstrated. In Section 5, the revised GK-SS method is applied to the performance optimization problem of the SG level control system. Based on the experimental simulation results, a comprehensive discussion was carried out. Finally, the conclusions are summarized.

\section{Performance Optimization of the Steam Generator Level Control System}

The general structure of the SG level control system in a pressurized water reactor (PWR) can be summarized as shown in Figure 1. According to the PWR commissioning practice, the main goal of the steam generator level control is to maintain the steam generator water level around a preset setpoint with the least power plant commissioning cost. To achieve the goal, the tuning process of the control system should be optimized. 


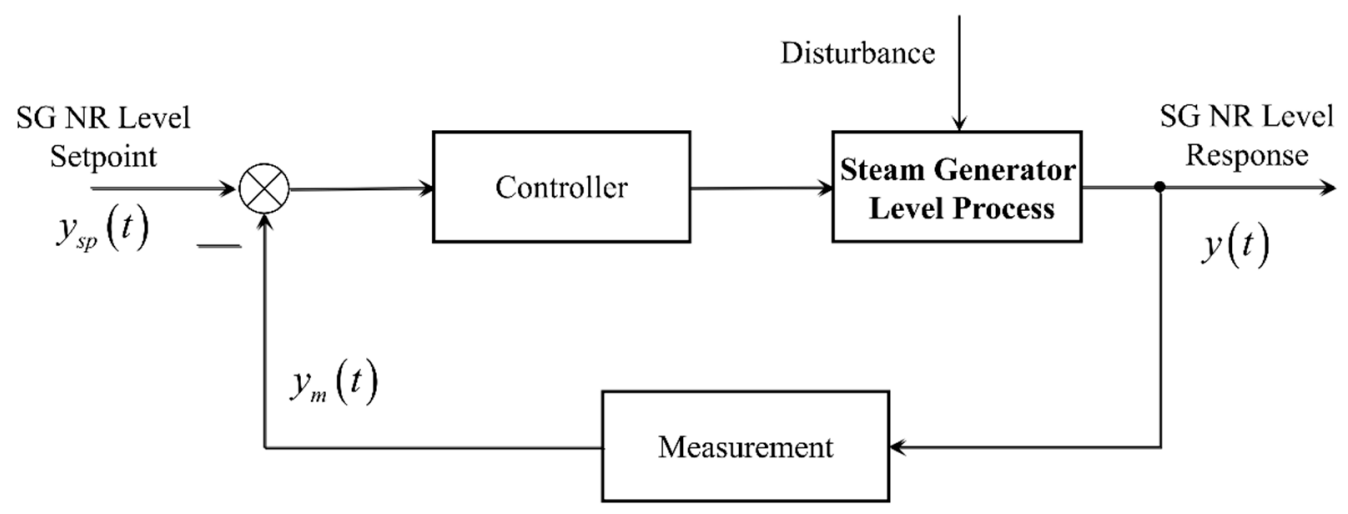

Figure 1. General structure of the steam generator (SG) level control system.

For a PWR plant, once the manufacturing of the steam generator and the control structure of the level controller have been fixed, the control performance of the SG level control system will mainly depend on the controller parameters. There is a close relationship between the control performance and the controller parameters. Without loss of generality, the mathematical relationship between the level control performance and the corresponding controller parameters could be expressed abstractly as below:

$$
\operatorname{Perf}=f(X)+e, \quad X=\left[x_{1}, \cdots x_{n}\right]^{T}
$$

where Perf represents the performance index to be optimized, $X$ is an $n$-dimensional vector representing the SG controller parameters, and $x_{i}(i=1, \cdots, n)$ is the $i$ th element of $X, n$ is the dimension of the parameters, $e$ represents the overall process performance uncertainty, $f(x)$ is the theoretical function between the performance and the controller parameters. However, due to the high complexity and nonlinearity of the SG level process, the above function is unable to obtain theoretically.

The performance optimization of the steam generator level control is, therefore, converted to tune the controller parameters and search for the optimal settings. The mathematical formula of the optimization problem can be expressed as below:

$$
\begin{aligned}
& \max _{X} \operatorname{Perf} \\
& \text { s.t. } X \in \Omega
\end{aligned}
$$

where $\Omega$ is the feasible region of the controller parameters. The schematic of the closed-loop optimization process based on data-driven optimization can be summarized in Figure 2.

To construct the closed-loop optimization scheme, it is necessary to find a suitable way to evaluate the control performance of the steam generator level control system. For a control system, the regulation performance is often expressed by the control error obtained under certain disturbances. Typical step load change can be chosen as the test scenario. Typical control evaluation index can be expressed as below:

$$
I=\int_{0}^{T_{t s}} t^{n}|e(t)|^{m} d t
$$

where the error is defined as $e=y_{s p}-y(t), y_{s p}$ is the target and $y(t)$ is the actual level response. $T_{t s}$ is a preset time zone for the evaluation of transient performance of the system. $n$ and $m$ are the index parameters, respectively. In this study, the Integral of Time multiply by Absolute Error (ITAE) index was chosen. The ITAE is an index widely used in control performance evaluation with $n=1, m=1$ in Equation (3). As the magnitude of the ITAE is huge, the logarithm of the ITAE was used to represent the control performance. According to the characteristics of the ITAE, the smaller the ITAE index, the better the control performance. 


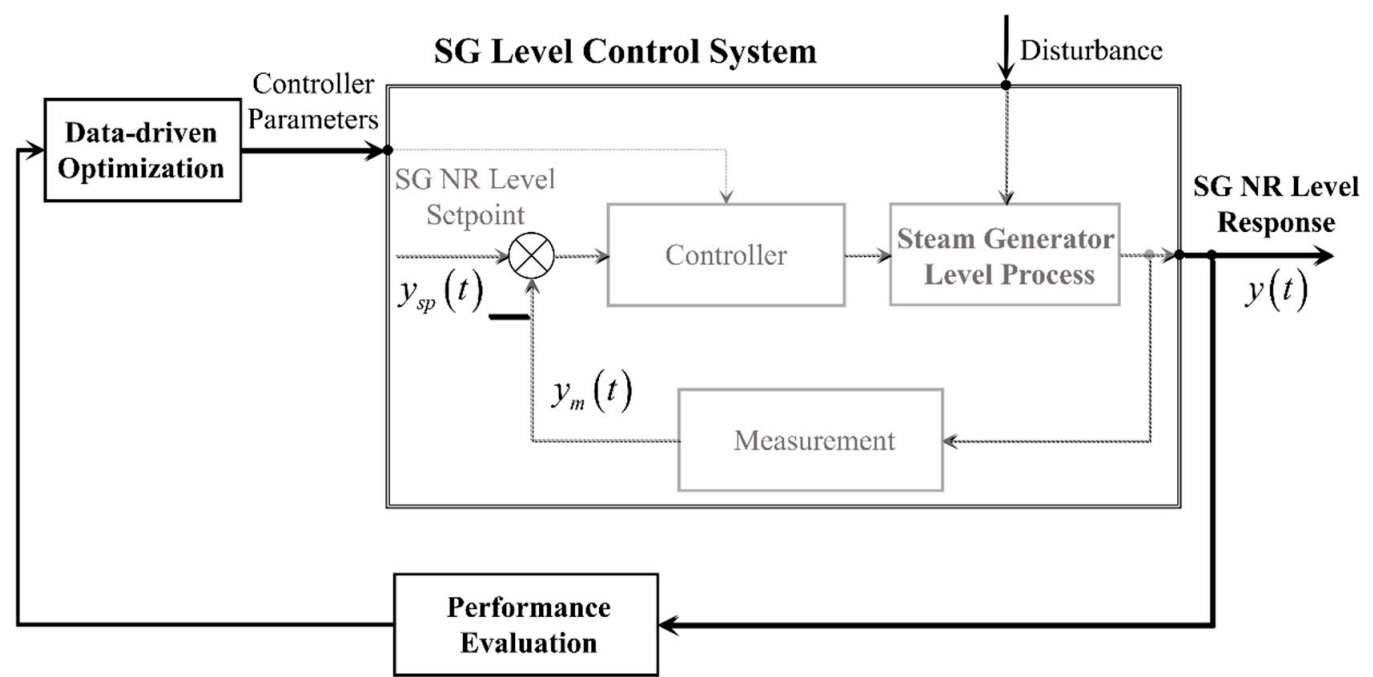

Figure 2. Schematics of the data-driven optimization for the SG level control.

With the above evaluation index, the controller performance optimization problem can be expressed as below:

$$
\begin{aligned}
& \min _{X}-\operatorname{Perf} \approx I=\int_{0}^{T_{t s}} t|e(t)| d t \\
& \text { s.t. } X \in \Omega
\end{aligned}
$$

The performance is approximately represented by the evaluation index.

Furthermore, in the PWR commissioning practice, the efficiency of the above optimization is a critical concern. The tuning process should be done as quickly as possible to reduce the commissioning cost and reduce its adverse effects on the plant. A new efficiency index, Optimization Efficiency index (OE), is defined. As a comprehensive efficiency index, it may integrate many aspects, such as experimental cost, time cost, labor cost and performance control deviation et al. Based on the OE index, the performance optimization may be reconstructed as a bi-level optimization problem. The mathematical formulation is as below:

$$
\begin{aligned}
& \max _{\text {OptMethods }} O E \\
& \text { s.t. } \quad\left\{\begin{array}{l}
\min _{X}-\operatorname{Perf} \approx I=\int_{0}^{T_{t s}} t|e(t)| d t \\
\text { s.t. } X \in \Omega
\end{array}\right\}_{\text {OptMethods }}
\end{aligned}
$$

where OptMethods is the available data-driven methods set, which satisfies the optimization framework in Figure 2. The key in this study is to search for a suitable method with high efficiency. Considering the plant commissioning practice, only the experimental cost was included in the OE. A revised simplex search-based model-free optimization method was thus proposed.

\section{Revised Simplex Search-Based Data-Driven Optimization}

\subsection{Performance Optimization via Data-Driven Optimization}

According to the characteristics of the optimization problem in Equation (2), the modelfree optimization methods could be incorporated for the optimization. The schematics of the model-free optimization are shown in Figure 3. These methods usually achieve their goal by pure historical iterative data. In the framework of the model-free optimization, the data-driven optimization mechanism will iteratively adjust the parameters of the control system to change the performance of the SG liquid level control system dynamically. The optimization iteration process is a batch-by-batch process. The efficiency of the tuning process will mainly depend on the optimization methods. This study adopts the simplex 
search method as the base method for its high efficiency in nonlinear system optimization. It is a direct search method without explicit gradient information.

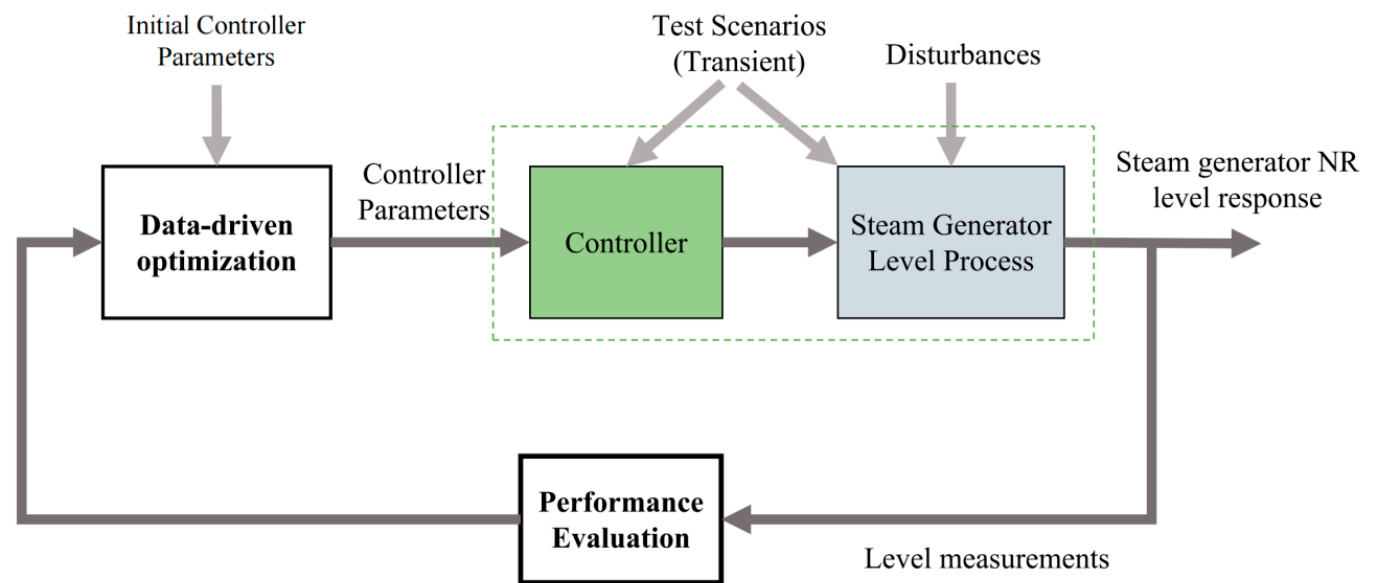

Figure 3. Framework of the model-free optimization (MFO).

However, the optimization cost is critical for a PWR plant's commissioning, and the simplex search method's efficiency should be further improved. Hence, the feasible directions for the performance efficiency promotion were analyzed as below. Figure 4 demonstrates a typical optimization trajectory under the traditional simplex search method. To promote the performance efficiency of the optimization method, the possibilities for the promotion were decomposed into two dimensions. For the first dimension, the optimization could be improved to achieve a rapid descent in the first stage of the optimization. If the optimization algorithms could be revised to accelerate its descent rate, the efficiency would be improved. For the second dimension, as a data-driven optimization method, the method usually cannot appropriately stop the optimization progress immediately. Thus, the optimization often costs more on iterations with only a minor achievement. The minor achievement is intolerable for the vast cost due to the additional iterations. Hence, if the method could terminate timely and intelligently when the optimization progress traps into the stagnation stage, the costs on the optimization could be reduced, and the efficiency thus could be promoted.
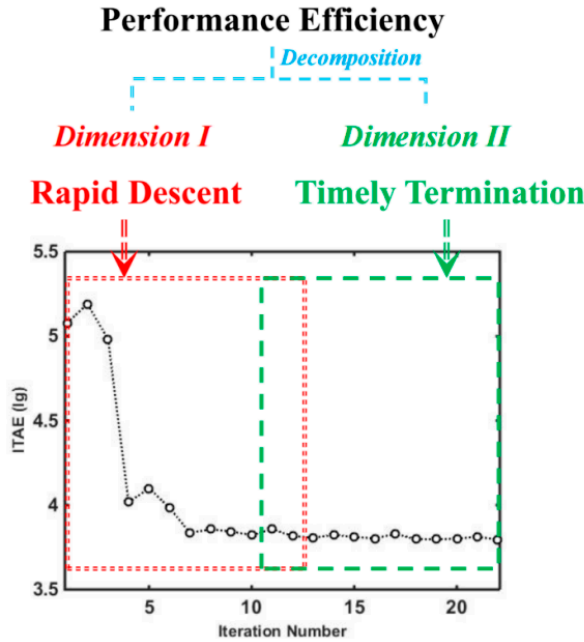

Figure 4. Decomposition of the directions for the performance efficiency promotion.

This study investigated both research dimensions for the simplex search-based modelfree optimization. Generally, as shown in Figure 5, the knowledge-guided mechanisms 
consist of two dimensions and three aspects. In Dimension I, considering that in the iterative process of performance optimization, the optimization process will generate a series of historical iterative process information containing specific process knowledge, in the traditional MFO methods, this kind of information is not fully utilized. In this study, the revised simplex search method may adopt the historical iteration points generated during optimization to adjust the search descent rate. Two aspects were concerned. The first aspect is the search direction of the optimization, with the second aspect being the step size of the search operation. The detailed mechanisms will be demonstrated in the following subsection. In Dimension II, the information of historical iteration points will be incorporated to form a historical sequence, and the optimization progress will be monitored and evaluated based on the sequence. Hence, the optimization progress could be interrupted timely when the method detects itself falling into the stagnation stage.

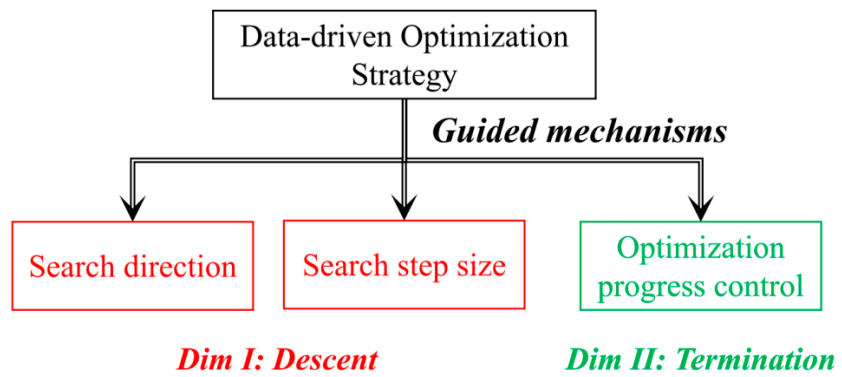

Figure 5. Historical information-guided mechanisms for the data-driven optimization.

From the idea of knowledge-informed optimization fused by iterative knowledge during the optimization, a revised simplex search-based model-free optimization based on the historical iteration information was formulated, and a feasible implementation scheme was put forward.

\subsection{Revised Simplex Search Strategy}

The simplex search method is a gradient-free method that relies only on the function evaluations [30]. Our team firstly proposed the initial revised simplex search method (GK-SS) to promote the efficiency of the quality control of the medium voltage insulator [27]. In this study, this method was transferred and revised further to adapt to the new optimization occasion of the controller parameters optimization. Furthermore, the GK-SS was reorganized based on the above ideas. The modifications for the traditional simplex search were reorganized into two parts, representing different mechanisms on efficiency promotion.

\subsubsection{Procedure of the Traditional Simplex Search Method}

The revised GK-SS was built based on the traditional Nelder-Mead method. The procedure of the method is as follows (More detailed procedure may refer to [27]):

Step 1: Methodology initialization.

The coefficients of the methodology, $\{\alpha, \beta, \gamma, \delta\}$, and the initial conditions $X_{1}$, are determined in this stage. The initial conditions will be scaled into a normalized range and represented as $\bar{X}_{1}$.

Step 2: Initial simplex construction.

The initial simplex is constructed obeying the following rule:

$$
\left\{\begin{array}{ll}
\bar{X}_{k+1}=\bar{X}_{1}+\tau e_{k}, & \bar{X}_{1, k} \leq 50 \\
\bar{X}_{k+1}=\bar{X}_{1}-\tau e_{k}, & \bar{X}_{1, k}>50
\end{array}, V_{k+1}^{s}=\bar{X}_{k+1}, k=1, \cdots, n\right.
$$

where $e_{k}$ is a special column vector with 1 in the $k$ th element and zeros in the other elements, $\bar{X}_{k+1}$ is the $(k+1)$ th iteration point, and $\tau$ represents the perturbation coefficient. With the construction, $n$ successive iteration points are generated based on the initial point, and the 
initial simplex is formed as $V^{s}=\left\{V_{1}^{s}, V_{2}^{s}, \cdots, V_{n+1}^{s}\right\}$ based on the $n+1$ points. $s$ is the simplex iteration number with its initial value set to 0 . The corresponding performance response ITAE vector $F^{s}=\left\{F_{1}^{s}, F_{2}^{s}, \cdots F_{n+1}^{s}\right\}$ is garnered by a series of experiments.

Step 3: Simplex Sorting.

The current simplex will be sorted according to their corresponding performance index $F^{s}$. The sorted simplex will be denoted as $V^{s *}$ with the following relation:

$$
F_{1}^{s *} \leq F_{2}^{s *} \leq \cdots F_{n}^{s *} \leq F_{n+1}^{s *}
$$

Thus, $V_{1}^{s *}$ is the vertex with the best response (with the smallest ITAE quality), $V_{n}^{s *}$ is the vertex with the next-to-the-worst response, $V_{n+1}^{s *}$ is the vertex with the worst response. The simplex iteration number will be updated by $s=s+1$.

Step 4: Reflection.

The reflection point $V_{r e f}^{s}$ will be generated as below:

$$
V_{r e f}^{s}=(1+\alpha) V_{c}^{s}-\alpha V_{n+1}^{s *}
$$

The centroid of all the vertices except $V_{n+1}^{s *}$ is expressed as:

$$
V_{c}^{s}=\left(\sum_{i=1}^{n} V_{i}^{s *}\right) / n
$$

Conduct the experiments to evaluate the ITAE performance index $F_{r e f}^{s}$ at the reflection point. If $F_{r e f}^{s}<F_{1}^{s *}$, the expansion operation should be executed; thus, the procedure goes to Step 5. If $F_{1}^{s *} \leq F_{r e f}^{s} \leq F_{n}^{s *}$, the procedure goes to Step 8. If $F_{r e f}^{s}>F_{n}^{s *}$, the contraction operation should be conducted, the procedure goes to Step 6 .

Step 5: Expansion.

The expansion point $V_{\exp }^{s}$ is generated with the following expansion rule:

$$
V_{\exp }^{s}=(1-\gamma) V_{c}^{s}+\gamma V_{r e f}^{s}
$$

The experiment will be conducted and the ITAE performance index $F_{\exp }^{s}$ would be evaluated. If $F_{\exp }^{s} \leq F_{r e f}^{s}$, the expansion is successful, $V_{n+1}^{s *}$ would be replaced by $V_{\exp }^{s}$. Otherwise, the worst vertex with the highest ITAE would be substituted by the reflection point. At last, the procedure goes to Step 8.

Step 6: Contraction.

The contraction point $V_{c t}^{s}$ could be calculated as below:

$$
V_{c t}^{s}=(1-\beta) V_{c}^{s}+\beta V_{\max / r e f}^{s}
$$

where $V_{\max / r e f}^{s}$ is the reference point for the contraction. The contraction operation consists of two modes: (i) inside contraction, (ii) outside contraction. The choice of the reference $V_{\max / r e f}^{s}$ depends on the contraction mode.

Case 1: Inside contraction.

When $F_{r e f}^{s} \geq F_{n+1}^{k *}$, let $V_{\max / r e f}^{s}=V_{n+1}^{s *}, F_{\max / r e f}^{s}=F_{n+1}^{s *}$; conduct the experiments to evaluate the ITAE index $F_{c t}^{s}$. If $F_{c t}^{s} \leq F_{n+1}^{s *}$, the contraction is accepted.

Case 2: Outside contraction.

When $F_{n}^{k *} \leq Y_{\text {ref }} \leq F_{n+1}^{k *}$, let $V_{\max / r e f}^{s}=V_{r e f}^{s}, F_{\max / r e f}^{s}=F_{r e f}^{s} ;$ conduct the experiments to evaluate the ITAE index $F_{c t}^{s}$. If $F_{c t}^{s} \leq F_{r e f}^{s}$, the contraction is accepted.

If the contraction is accepted, replace $V_{n+1}^{s *}$ with $V_{c t}^{s}$ and go to Step 8. Otherwise, if the contraction is refused, go to Step 7 for a shrink operation.

Step 7: Shrink.

The shrink operation is generated as below:

$$
V_{i}^{s *}=(1-\delta) V_{1}^{s *}+\delta V_{i}^{s *}, \quad i=2, \cdots, n+1
$$


The best vertex will be retained. All the vertices, except the best one, are updated according to Equation (12). The ITAE performance indices of the updated vertices will be measured and updated.

Step 8: Termination.

The termination module will judge the termination rule based on the revised iteration termination control strategy.

The traditional simplex-search method was revised according to the knowledgeinformed idea in this study. A data-driven method, the GK-SS, was reformulated. The GK-SS deals with the act of optimization as an entirety. The two key modifications are detailed in the following subsections, respectively.

\subsubsection{Search Mechanism Modification}

The search mechanism was redesigned from the idea of Dimension I of the efficiency promotion. The revised mechanism was conducive to the rapid descent of the optimization process. To build a more efficient knowledge-informed mechanism, the traditional SS was reviewed. The essence of the SS's principle was further revealed and strengthened. The SS is reshaped to construct a new quantity-quasi-gradient estimation from the above perspective. With this new quantity, the quasi-historical gradient estimation information can be recorded and utilized to improve the accuracy of the quasi-gradient estimations for the simplex search method. Once the accuracy of the gradient estimations improved, the simplex-search method's efficiency would be enhanced accordingly. Figure 6. demonstrates the schematic diagram of the quasi-gradient estimation compensation mechanism of the GK-SS. It can be seen from the figure that the compensated search direction will approach the theoretical steepest search direction with the help of the historical quasi-gradient estimations.

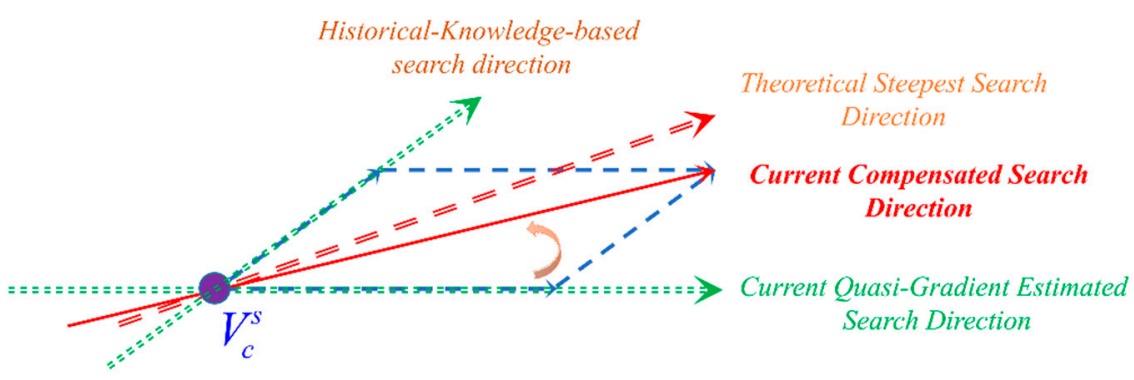

Figure 6. Sketch of the compensation mechanism [27].

Hence, a specific simplex search mechanism was proposed, which incorporates the historical quasi gradient estimations to facilitate the optimization. The quasi-gradient estimation is expressed as follows:

$$
\widetilde{G}_{c}^{s}=V_{n+1}^{s *}-V_{c}^{s}
$$

where $\widetilde{G}_{c}^{s}$ is the estimated quasi-gradient for current simplex (EGCS) at the sth simplex.

The iteration strategy of the simplex search method is represented as below:

$$
V_{\text {vertex_new }}^{s}=V_{c}^{s}-\xi \widetilde{G}_{c}^{s}
$$

where $\xi$ is the estimated step size of the method, which is determined by the status of the current simplex.

To integrate the historical knowledge to compensate the gradient estimation accuracy at the current simplex, the compensated composite gradient for the current simplex (CCG) is defined as follows:

$$
\hat{G}_{c}^{s}=\rho_{s} \hat{G}_{c}^{s-1}+\left(1-\rho_{s}\right) \widetilde{G}_{c}^{s}, \hat{G}_{c}^{1}=\widetilde{G}_{c}^{1}
$$

where $\hat{G}_{c}^{s}$ is the CCG at the sth simplex, $\hat{G}_{c}^{s-1}$ is the CCG at the (s-1)th simplex, $\hat{G}_{c}^{1}$ is the CCG at the 1st simplex. $\rho_{s}$ is the gradient compensation coefficient at the sth simplex. 
The gradient compensation coefficient updating rule is as below:

$$
\rho_{s}=\rho_{F}-\frac{\Delta \rho_{\text {init }}}{s^{\tau}}
$$

where $\rho_{F}$ is an upper limit that a CCG approached, which is set to 0.5 by default, $\Delta \rho_{\text {init }}$ is the initial deviation between $\rho_{F}$ and its lower limit, which is set to 0.2 by default, and $\tau$ is an exponential coefficient.

The original reflection operation, Equation (8), should be substituted as follows:

$$
\bar{V}_{r e f}^{s}=V_{c}^{s}-\alpha \hat{G}_{c}^{s}
$$

The expansion operation would be revised as below:

$$
\bar{V}_{\exp }^{s}=(1-\gamma) V_{c}^{s}+\gamma \bar{V}_{r e f}^{s}=V_{c}^{s}-\alpha \gamma \hat{G}_{c}^{s}
$$

The outside contraction is as follows:

$$
\bar{V}_{\text {oct }}^{s}=(1-\beta) V_{c}^{s}+\beta \bar{V}_{\text {ref }}^{s}=V_{c}^{s}-\alpha \beta \hat{G}_{c}^{s}
$$

The inside contraction is as below:

$$
\bar{V}_{i c t}^{s}=(1-\beta) V_{c}^{s}+\beta \bar{V}_{n+1}^{s}=V_{c}^{s}+\beta \hat{G}_{c}^{s}
$$

The quasi-gradient information is incorporated with the above modification, and the revised mechanism is formulated. The different search mechanisms of the traditional simplex-search and the revised method are illustrated and compared in Figure 7. The revised GK-SS method is a typical knowledge-informed optimization strategy that utilizes the historical quasi-gradient estimations to compensate for the search direction at each simplex iteration. In a statistical sense, this strategy could significantly accelerate the search rate of the optimization.

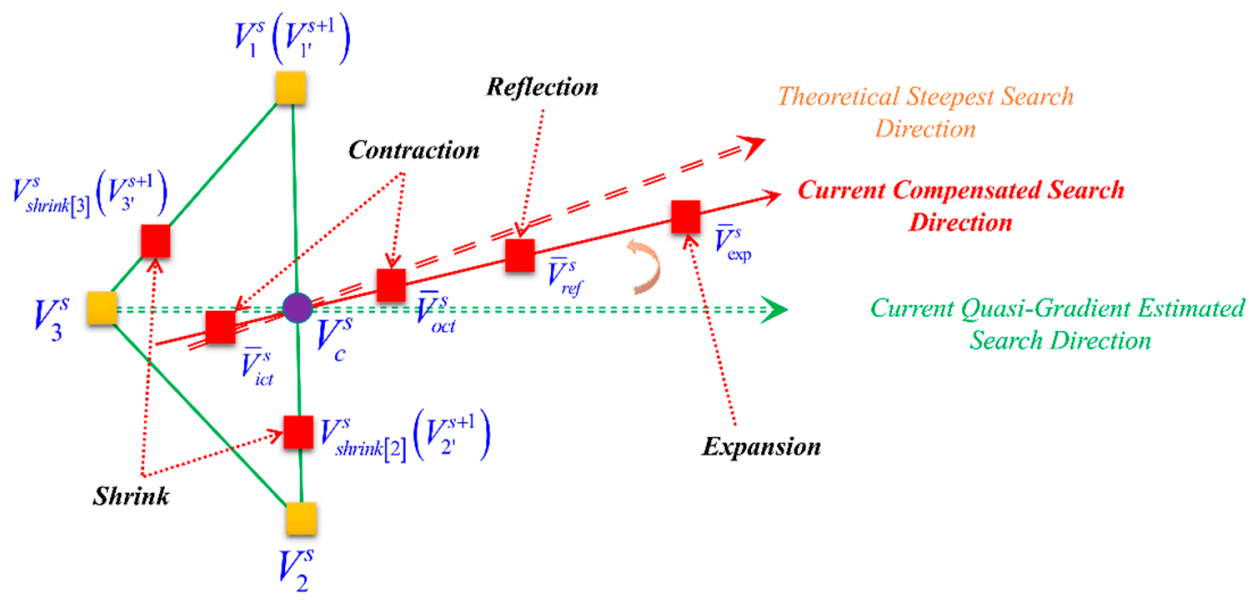

Figure 7. Optimization mechanisms of the knowledge-informed simplex search based on historical gradient approximations (GK-SS) illustrated in a two-dimensional occasion [27].

\subsubsection{Iteration Termination Control Modification}

Data-driven optimization relies on commissioning experiments. Hence, from Dimension II, the efficiency may be promoted through timely termination of the optimization progress. Usually, when the optimization progress goes into the stagnation zone, the improvement in the optimization becomes significantly slight. Too many experiments may only lead to trivial promotion. To reduce the commissioning costs, proper termination criteria should be developed. If the optimization progress could be monitored, the optimization method may construct a proper termination rule to timely terminate the optimization. In 
this study, iteration termination control strategy was designed to take advantage of the achievement estimation of the optimization process to discover the proper time to terminate the optimization progress [23].

Figure 8 shows the framework of the revised iteration termination strategy. The procedure can be divided into seven steps as follows:

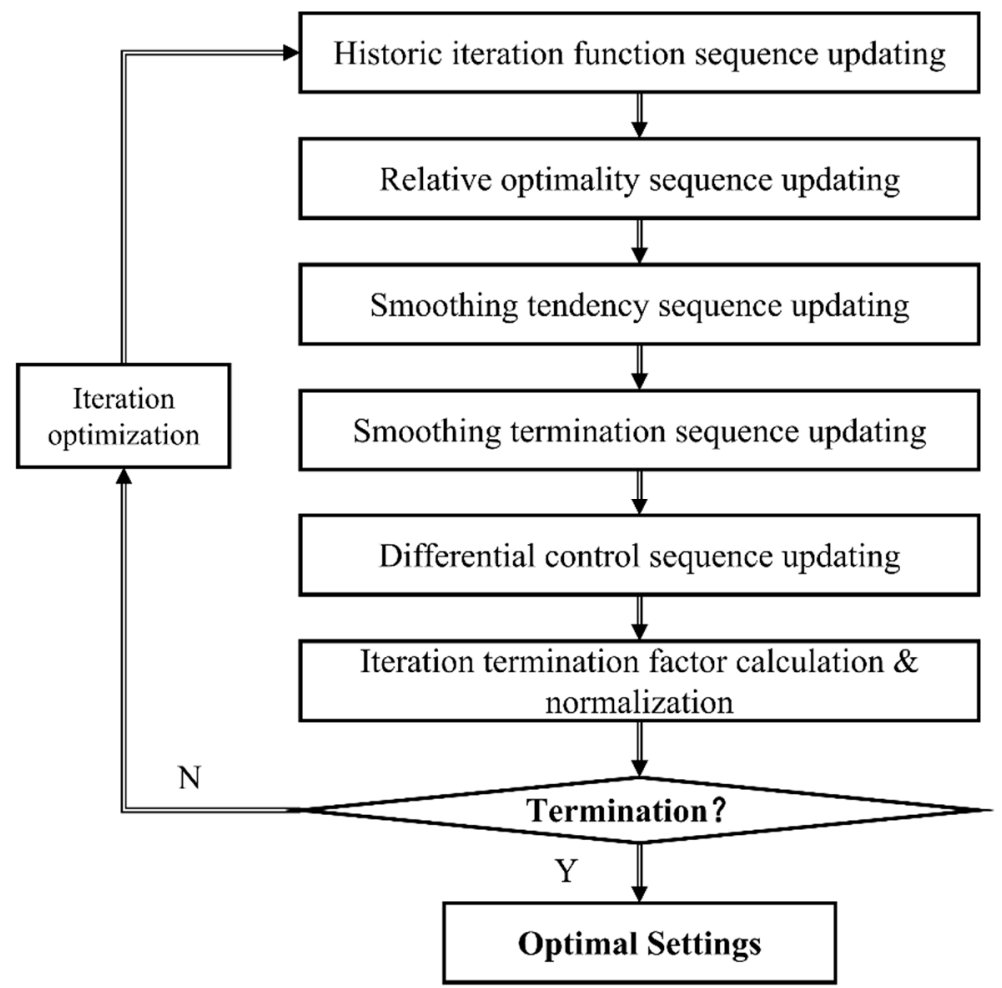

Figure 8. Framework of the Iteration Termination Control.

Step 1: Historic iteration function sequence updating.

Historic iteration function sequence, $S_{H}$, is a self-increasing sequence storing the function evaluations of all the historic iteration points. When a new iteration point is generated, and the corresponding experiment is conducted, the evaluation of the ITAE index will be recorded. Then, the Sequence $S_{H}$ will grow with the new evaluation.

Step 2: Relative optimality sequence updating.

In this stage, the historic iteration function sequence will be sorted according to the magnitude of the control performance index. The best index is gained and recorded iteratively, and a relative optimality sequence, $S_{R O}$, is updated sequentially with the current best index.

$$
S_{R O}(i)=\min \left(S_{H}\right)
$$

where $i$ is the current count number of the iterations.

Step 3: Smoothing tendency sequence updating.

The relative optimality sequence is further smoothed by a moving average method. Then, the tendency sequence $S_{S T}$ is obtained as below:

$$
S_{S T}(i)=\left\{\begin{array}{cl}
\frac{1}{i} \sum_{k=1}^{i} S_{R O}(k), & i \in[1, \smile(n+1)) ; \\
\frac{1}{(n+1)} \sum_{k=i-\lambda(n+1)-1}^{i} S_{R O}(k), & i \in[\lambda(n+1),+\infty) .
\end{array}\right.
$$

where $n$ is the parameters dimension, $\lambda$ is the smoothing coefficient.

Step 4: Smoothing termination sequence updating. 
To obtain a more meaningful sequence, another smooth action is conducted further. The termination sequence $S_{T M}$, to be utilized for the termination control, is generated. It is a monotonically decreasing sequence. Its formulation rule is defined as below:

$$
S_{T M}(i)=\left\{\begin{array}{cl}
\frac{1}{i} \sum_{k=1}^{i} S_{S T}(k), & i \in[1, \eta(n+1)) ; \\
\frac{1}{\eta(n+1)} \sum_{k=i}^{i-\eta(n+1)+1} S_{S T}(k), & i \in[\eta(n+1),+\infty) .
\end{array}\right.
$$

Step 5: Differential control sequence updating.

The differential control sequence $\Delta S_{T M}$ is formulated as below:

$$
\Delta S_{T M}(i)=\left\{\begin{array}{l}
1, i \in[1, \eta(n+1)] \\
S_{T M}(i)-S_{T M}(i-\eta(n+1)+1), i \in[\eta(n+1),+\infty]
\end{array}\right.
$$

Step 6: Iteration termination factor calculation \& normalization.

To evaluate the relative progress of the optimization, the termination factor $\xi(i)$ is defined:

$$
\xi(i)=\left\{\begin{array}{l}
0, \\
\frac{\Delta S_{T M}(i)}{S_{T M}(i)}
\end{array}\right.
$$

It indicates the ratio of the control performance improvement at the current point.

However, the numerical range of this factor is too broad, which is highly related to the specific problems and the optimization process at different times, so it may appear at different scales when used to judge the status of the optimization process. In fact, in the actual operation of the SG level control performance optimization, the termination factor may vary quite sharply. It may deteriorate the function of the iteration termination control. Hence, a further normalization mechanism was invented to cope with this challenge.

Considering the consistency of the judgment factor, the factors should be reformulated. Each calculated factor is added to form a sequence; thus, a termination factor sequence $S_{\xi}$ is generated.

A new normalized sequence is defined as below:

$$
\varsigma(k)=\frac{\xi(k)-\min \left(S_{\xi}\right)}{\max \left(S_{\xi}\right)-\min \left(S_{\xi}\right)}, k=1, \cdots, i .
$$

At each iteration, every element of the entire sequence will be recalculated, and the sequence is scrolled.

Step 7: Termination criteria judgment.

When the current judgment factor is small enough, i.e., $\varsigma(i)<\varsigma_{T}$ ( $\varsigma_{T}$ is the tolerance), the progress at the current iteration is so slight that the termination criteria may be satisfied. However, to avoid the prematurity of optimization, further verification should be conducted. The iteration terminate rule is defined as:

$$
\left(\zeta(i)<\varsigma_{T}\right) \cap\left(\kappa=\kappa_{F}\right)
$$

where $\kappa_{F}$ is the repeating coefficient set by the engineers, $\kappa$ is a counter representing the number of successive iterations that satisfies the former tolerance.

When Equation (27) is satisfied, the controller parameters optimization process will be terminated. The optimal settings will be set on the controller for the SG level control.

The anatomy of the GK-SS, including the two modifications, is illustrated in Figure 9. The primary mechanism of the GK-SS uses knowledge of historical quasi-gradient information generated during the iteration of the simplices to predict more accurate search directions and uses the historical iteration function evaluations to monitor the optimization progress. The revised method leverages synergies across different mechanisms within 
the data-driven optimization strategy. With these two modifications, the efficiency of the optimization would be enhanced.

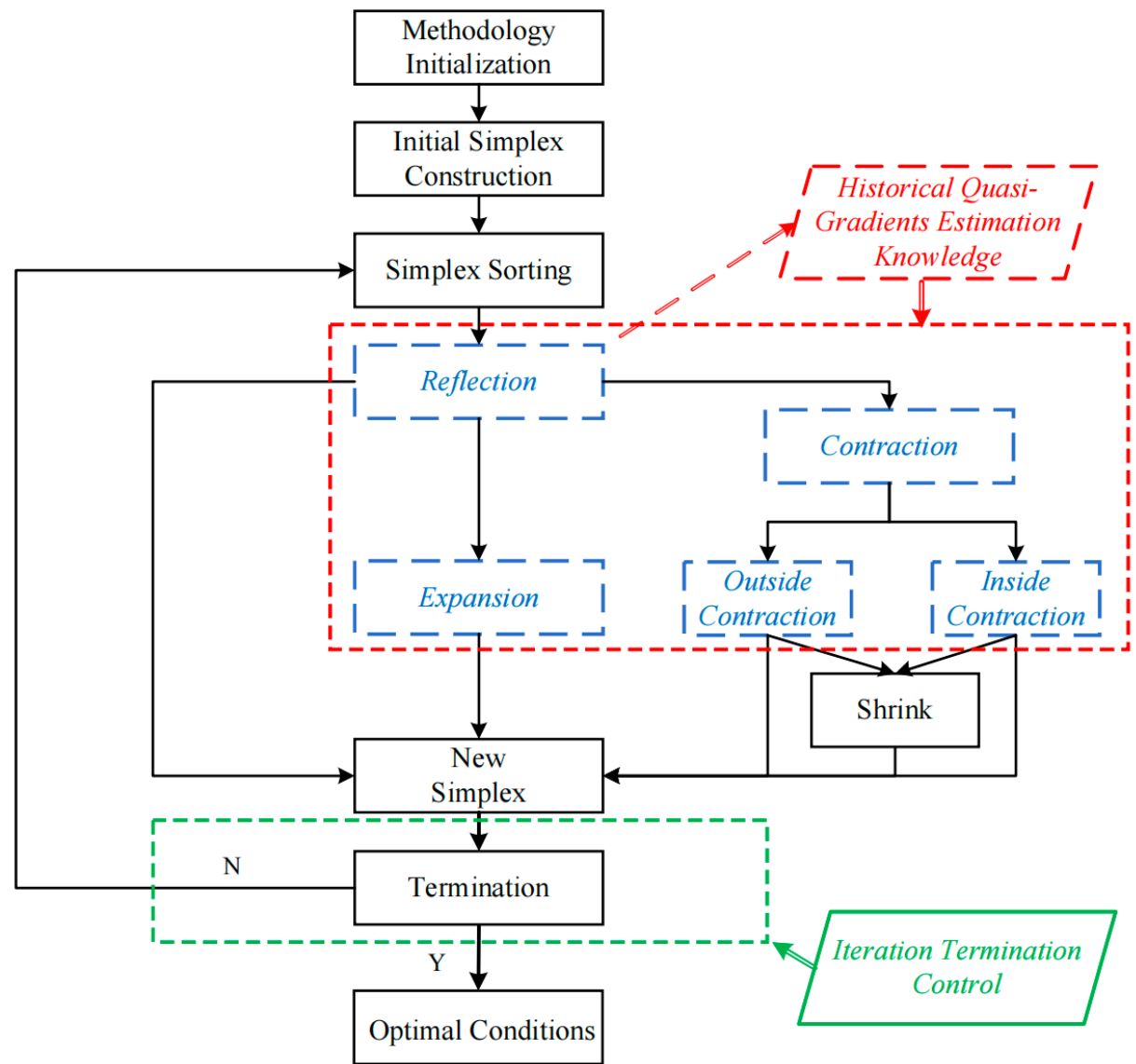

Figure 9. Anatomy of the revised GK-SS.

\section{Simulation Experimental Setup}

Considering the feasibility of verifying the proposed methodology's effectiveness and efficiency, a simulation platform for the SG level control system was established. The platform consists of the process model of the steam generator, the control scheme, and the controller parameters.

The general structure of a steam generator is illustrated in Figure 10. A highly complex and nonlinear system should adopt a suitable simplified model for the controller design. A widely used simplified steam generator level model was proposed by Irving et al. [2] and was adopted as the process model. The model captures the essential dynamics of the steam generator and is widely used for the simulation and control of the steam generator level process. The transfer function of the model is as follows:

$Y(s)=\frac{G_{1}}{s}\left(Q_{e}(s)-Q_{v}(s)\right)-\frac{G_{2}}{1+\tau_{2} s}\left(Q_{e}(s)-Q_{v}(s)\right)+\frac{G_{3}(s)}{\tau_{1}^{-2}+4 \pi T^{-2}+2 \tau_{1}^{-1} s+s^{2}} Q_{e}(s)$

where $Y(s)$ is the output of the model, which represents the narrow range water level of the steam generator. $Q_{e}(s)$ and $Q_{v}(s)$ are the inputs to the steam generator, where $Q_{e}(s)$ represents the feed water rate and $Q_{v}(s)$ is the steam rate. 


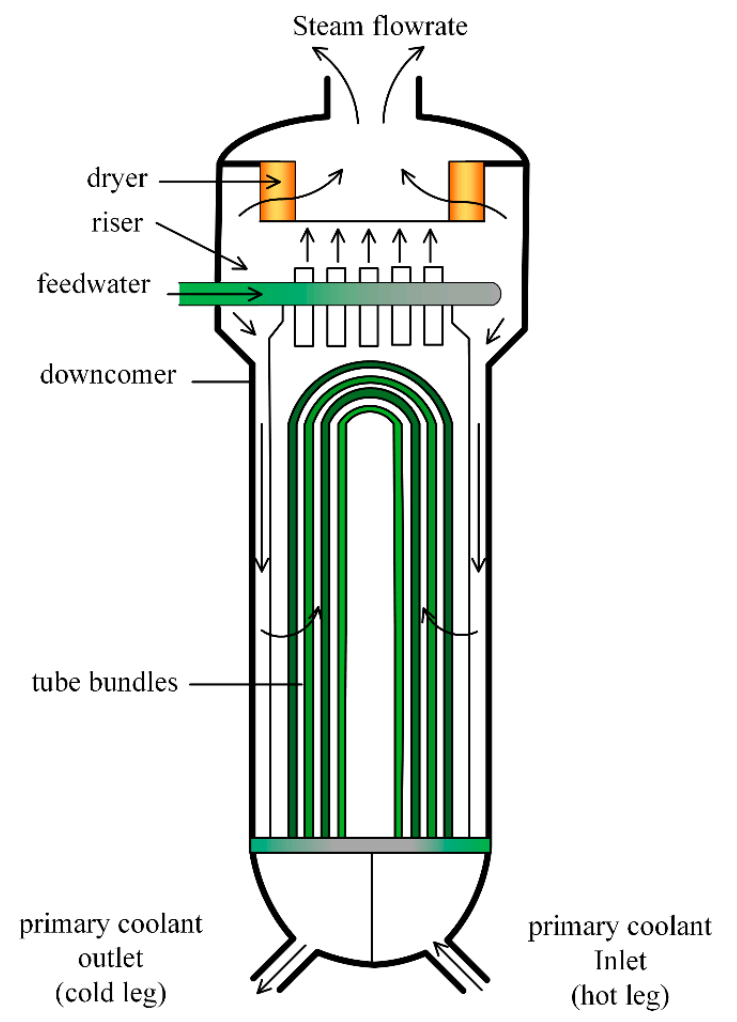

Figure 10. Structure of the U-type steam generator.

In Equation (28), the output is comprised of three terms. The first term represents the water level due to the total mass inventory in the steam generator. The second term represents the negative thermal effect of the "swell and shrinks" phenomenon. It produces an unstable zero, which accounts for the non-minimum phase behavior of the steam generator. The third term represents the mechanical oscillations caused by the feed water rate [31].

$G_{1}, G_{2}, G_{3}, \tau_{1}, \tau_{2}$, and $T$ are the parameters of the steam generator model. $G_{1}$ is the magnitude of the mass capacity effects, $G_{2}$ is the magnitude of the "swell and shrinks" phenomenon, and $G_{3}$ is the magnitude of the mechanical oscillation. $\tau_{1}, \tau_{2}$ are the damping time constants. $T$ is the period of the mechanical oscillation. All these parameters are highly related to the turbine load of the nuclear power plant. When the power level $P$ (\% Full Power, \% FP) varies, the parameters should be changed. To represent the SG with the full power range, the model parameters at different power levels have been identified from experimental data by Irving, E., and the identified parameters are given in Table 1.

Table 1. Parameters of the simplified steam generator model.

\begin{tabular}{cccccc}
\hline $\boldsymbol{P}(\boldsymbol{\%}$ FP) & $\mathbf{5}$ & $\mathbf{1 5}$ & $\mathbf{3 0}$ & $\mathbf{5 0}$ & $\mathbf{1 0 0}$ \\
\hline$G_{1}$ & 0.058 & 0.058 & 0.058 & 0.058 & 0.058 \\
$G_{2}$ & 9.63 & 4.46 & 1.83 & 1.05 & 0.47 \\
$G_{3}$ & 0.181 & 0.226 & 0.310 & 0.215 & 0.105 \\
$\tau_{1}$ & 41.9 & 26.3 & 43.4 & 34.8 & 28.6 \\
$\tau_{2}$ & 48.4 & 21.5 & 4.5 & 3.6 & 3.4 \\
$T$ & 119.6 & 60.5 & 17.7 & 14.2 & 11.7 \\
$Q_{v}(s)(\mathrm{kg} / \mathrm{s})$ & 57.4 & 180.8 & 381.7 & 660 & 1435 \\
\hline
\end{tabular}

A three-element control system with a cascade PID control scheme was adopted to achieve a suitable control target. The diagram of the control system is demonstrated in Figure 11. 


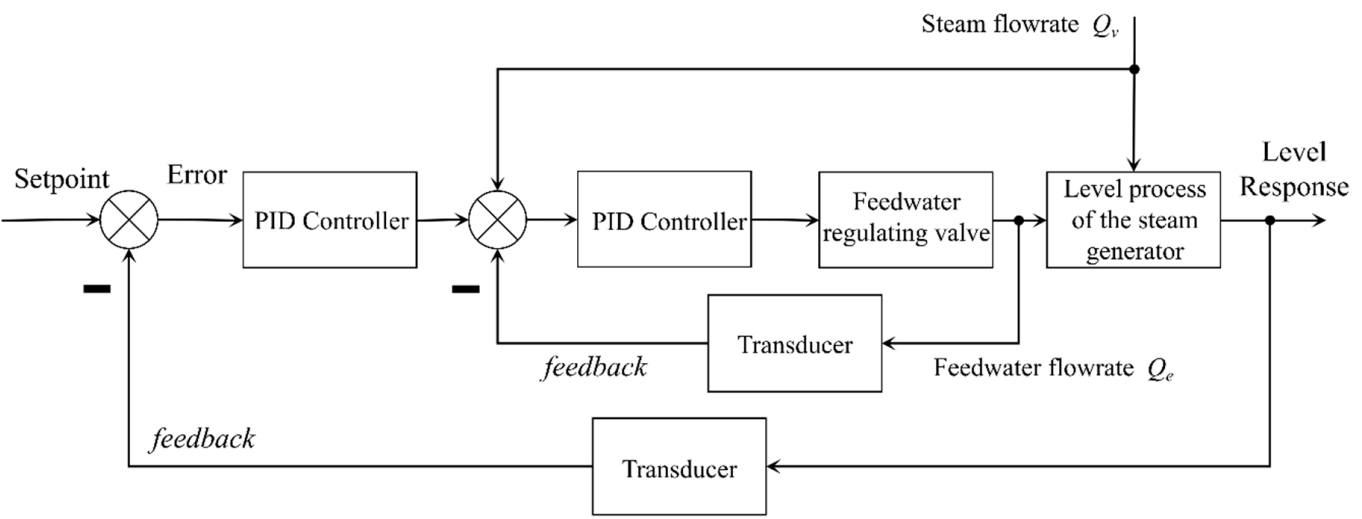

Figure 11. Cascade Proportional-Integral-Derivative (PID)-based control scheme for the SG level control.

This three-element scheme takes the feedwater flow as the internal disturbance, treats its signal as the second controlled variable, and regulates it through the vice-loop controller. Meanwhile, the scheme takes the steam flow as the external disturbance and adds it to the vice-loop controller in a feedforward way. With this architecture, the negative feedback of feedwater flow disturbance reduces the system's dynamics, while the feedforward of the steam flow weakens the phenomenon of "swell and shrinks" effects; thus, the stability and rapidity of the system operation could be improved.

However, the controller parameters of the cascade PID controller still have a significant impact on the performance of the steam generator control system. To further promote the performance of the steam generator control system, the controller parameters need to be improved.

The cascade PID scheme consists of two independent PID controllers: the principal regulator and the auxiliary regulator. Each PID controller has the control scheme as follows:

$$
G(s)=k_{P}+\frac{k_{I}}{s}+k_{D^{S}}
$$

where $k_{P}$ represents the proportion gain, $k_{I}$ represents the integral gain, $k_{D}$ represents the derivative gain.

As the process and the architecture of the control system have been determined, the system's performance will be mainly affected by the parameters of the two PID controllers. The PID parameters of the control system to be optimized can be defined as $X=\left[x_{1}, x_{2}, x_{3}, x_{4}, x_{5}, x_{6}\right]^{T}$. As a particular industrial process, the NPP should be kept stable during the PID parameters tuning process in the plant commission. Hence, the feasible region of the PID parameters, determined by the recommendation of the plant design specification and the engineers' experience, guarantee the system's stability. As a result, the performance optimization of the NPP control system is an optimization process on the premise of the stability of the control system. Therefore, the stability of the control system, as an implicit assumption, does not need to be further discussed in this paper. The feasible region of the PID parameters in this study is shown in Table 2.

Table 2. Feasible regions of the cascade Proportional-Integral-Derivative (PID) control system.

\begin{tabular}{cccc}
\hline Variable No. & Description & Low Limits & Upper Limits \\
\hline $\mathrm{x}_{1}$ & $k_{P}$ of the principal regulator & 0.077 & 0.3 \\
$\mathrm{x}_{2}$ & $k_{I}$ of the principal regulator & $2.3 \times 10^{-4}$ & $2.3 \times 10^{-3}$ \\
$\mathrm{x}_{3}$ & $k_{D}$ of the principal regulator & -0.6 & 2.65 \\
$\mathrm{x}_{4}$ & $k_{P}$ of the auxiliary regulator & 1 & 1.5 \\
$\mathrm{x}_{5}$ & $k_{I}$ of the auxiliary regulator & 0.3 & 0.8 \\
$\mathrm{x}_{6}$ & $k_{D}$ of the auxiliary regulator & 0 & 0.5 \\
\hline
\end{tabular}


The above platform was built based on Simulink ${ }^{\circledR}$ v2021a. To search for the optimal PID parameters, the proposed method was formulated and verified based on the simulation platform.

A typical transient condition was adopted to verify the method at the same conditions. For each simulation experiment, the nuclear power plant is firstly operated at the initial condition for $100 \mathrm{~s}$; then, the transient is actuated. After the actuation of the transient, the nuclear power plant model will run for $1100 \mathrm{~s}$ to ensure the plant return to a new steady state. The steam generator level response is recorded during the process, and the ITAE index is calculated after each simulation. The results can be analyzed in the next section.

\section{Results and Discussion}

The revised simplex search-based MFO (GK-SS-based MFO) was conducted on the above simulation platform. To verify the effectiveness of the improved method and its efficiency relative to the traditional method, two groups of tests, including the effectiveness test and the efficiency test, are designed, respectively, for the verification.

\subsection{Effectiveness Test}

Firstly, without loss of generality, a randomly initial point $\mathrm{x}_{1}=[0.077,0.0003,0.2$, $1,0.5,0]^{T}$ was selected. The steam generator model was operated at $100 \%$ FP. The same test condition was implemented on each iteration experiment in this test. The GK-SS was conducted, and the optimization results were recorded. The optimization trajectory represented by ITAE is demonstrated in Figure 12. It can be observed that the parameters tuning with a significant performance improvement had finished in a relatively limited iteration number. With the GK-SS's optimization process, the ITAE index continued to decline. The controller parameters were perturbed dynamically during the tuning process, iteration by iteration. The trajectories of the controller parameters can be seen in Figure 13. Finally, the optimized point achieved by the GK-SS-based MFO is $\mathrm{x}_{\text {opt }}=[0.26,0.0023,-0.4331,1.4883,0.8,0.3047]^{T}$. To show the performance differences between the initial guess $x_{1}$ and the optimized point $x_{o p t}$, the level responses under the two different settings are shown in Figure 14. From the figures, it can be seen clearly that a significant improvement was obtained from $\mathrm{x}_{1}$ to $\mathrm{x}_{\mathrm{opt}}$ with the help of the GK-SS.

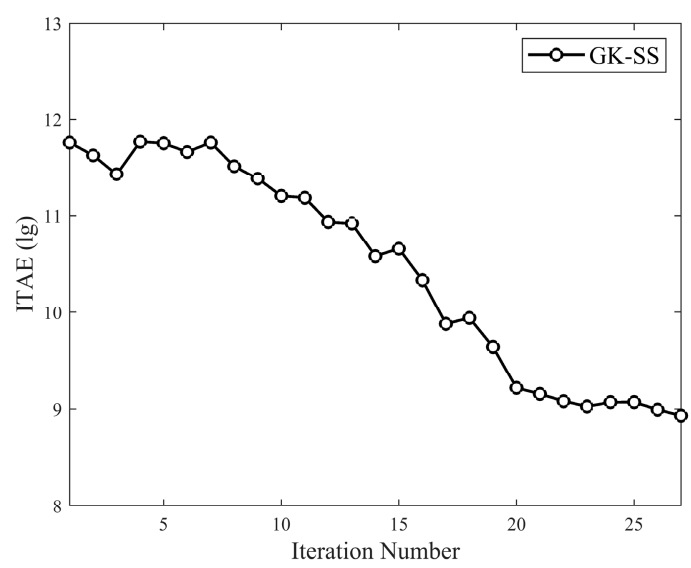

Figure 12. Iteration points trajectory of the revised simplex-search-based MFO.

The effectiveness of the iteration termination control was verified further. Another initial point was selected. The termination factor was recorded and plotted. The trajectory of the termination factor is demonstrated in Figure 15. It can be seen that the termination factor firstly went up with the optimization descending process, then the factor went down as it gradually entered the stagnation zone. The termination factor tolerance was set to 0.2. The optimization progress stopped in four successive iterations as the termination 
factor successively became smaller than the tolerance. A total of 24 iterations was spent on this test.

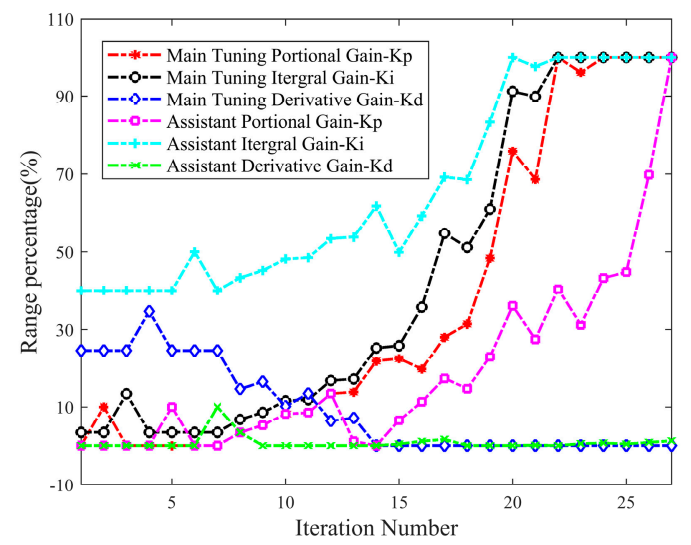

Figure 13. Trajectories of the controller parameters during the optimization.

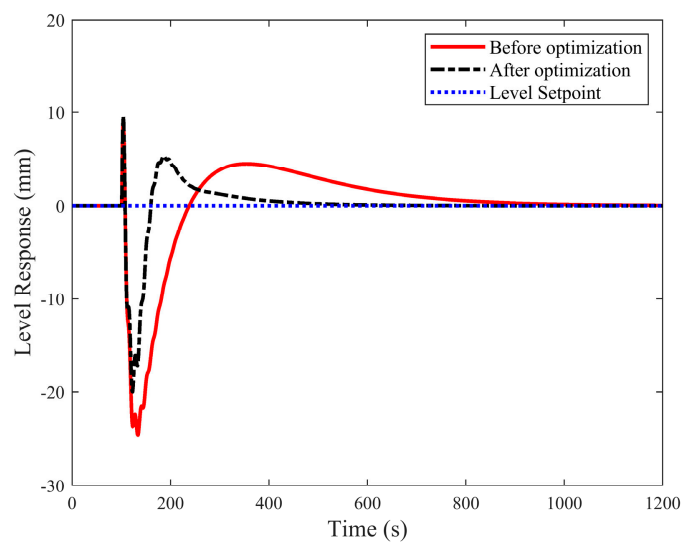

Figure 14. Level trajectories before and after the optimization.

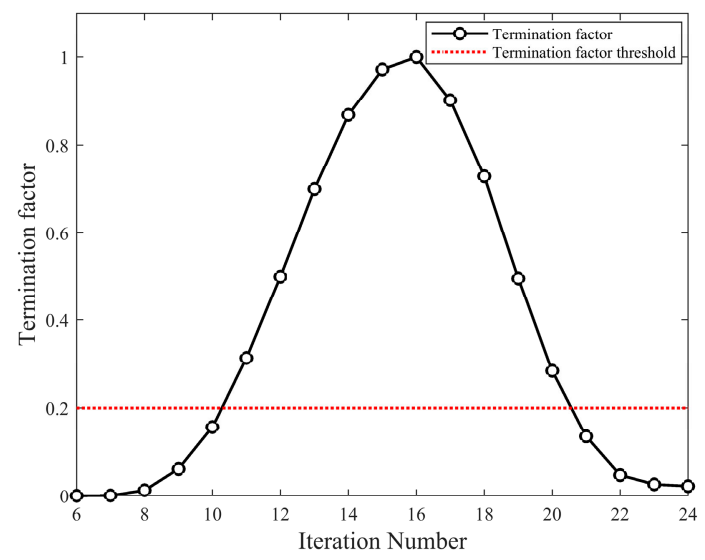

Figure 15. Trajectory of the termination factor.

To show the effectiveness from different initial points, three different initial points were chosen randomly. The optimization trajectories are demonstrated in Figure 16. All the optimization runs behaved similarly in the optimization tendency, in that the method was effective from different initial points. 


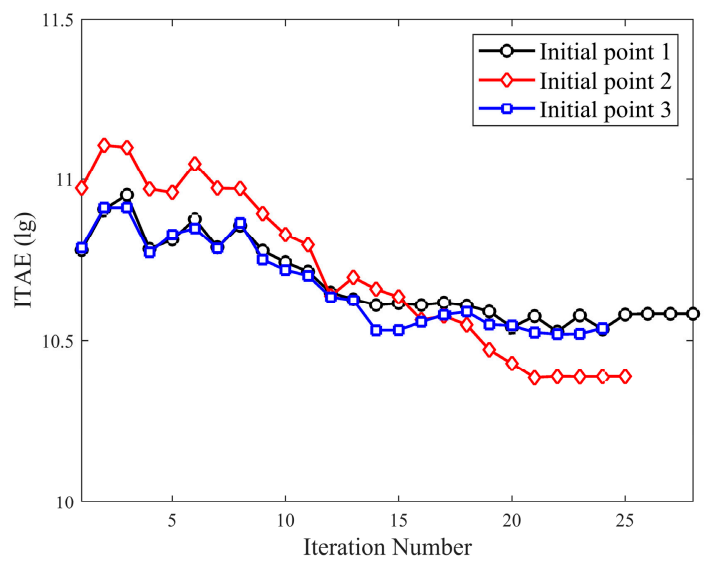

Figure 16. Optimization trajectories from different initial points.

To show the effectiveness of the GK-SS on different operation conditions, two operation scenarios under different power levels were selected-one case from the $100 \% \mathrm{FP}$, another one from the $50 \%$ FP. The optimization trajectories are shown in Figure 17. It can be seen clearly that the GK-SS behaved well under both scenarios.

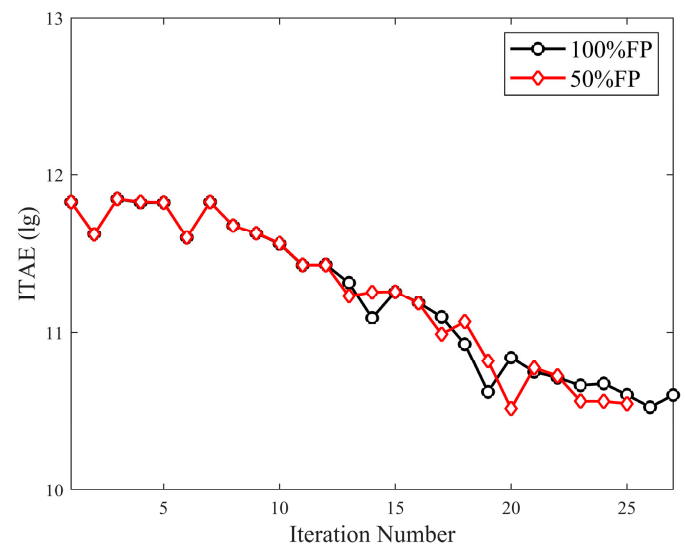

Figure 17. Level trajectories at the start and the end of the optimization.

According to the above tests, the effectiveness of the revised simplex-search-based MFO was clearly demonstrated.

\subsection{Efficiency Test}

From the above tests, the revised method, GK-SS, was showcased to be effective on the performance optimization of the SG level control system. However, the efficiency improvement of the GK-SS relative to the traditional simplex search method should be tested further. The same optimization test framework, a sequential Latin Hypercube Sampling (LHS) strategy, proposed in [17], was incorporated. In the test framework, for a single batch, the regular LHS is adopted, generating a sample of randomly distributed initial points. Then, to investigate the method's tendency with the enhancement of the sample, the same LHS operation is repeated sequentially. A repeated number of LHS tests were conducted to form a series of LHS sample tests to monitor the tendency. This kind of design of experiments (DOE) may cover enough initial points that it will provide a statistical result to show the relative efficiency of the GK-SS method.

In the test, the dimension of the process conditions was 6. Considering adopting a reasonable level for the controller parameters, each parameter was equally divided into ten levels. For a single LHS, ten independent sampling points were generated randomly. To evaluate the performance of an optimization run objectively, the iteration number of the transient process on performance optimization was selected as a critical index to show 
the efficiency of the method. The average iteration number was calculated to reflect the relative performance of the two methods on each batch. In this test, the batch number of the sequential design was set to 100, that is, 100 LHS batches with a total of 1000 optimization runs. This quantity of the randomly selected initial points could provide enough samples for the statistical analysis of the method's performance.

For comparison, both the GK-SS and the SS were implemented, respectively. All the experiments were carried out under identical test conditions, with only the difference in the initial points. The results of every LHS batch were recorded and observed. The dot diagram of the averaged iteration number for each LHS batch is shown in Figure 18a. It can be seen from the figure that the average iteration number per LHS batch varied considerably for both methods. However, it was observed that the iteration number of the SS was significantly greater than the GK-SS. From the perspective of statistical view, the accumulated averaged iteration number of the GK-SS is illustrated in Figure 18b. It can be seen from the figure that the accumulated average iteration number of the GK-SS was significantly decreased relative to the SS. The sequential LHS design provided an angle of view to observe the trend of the performance indices with the increase of independent initial points. Figure $18 \mathrm{~b}$ showed the accumulated average iteration number trajectories of the GKSS and the SS. In the initial LHS batches, due to the limited coverage of the total samples, it was shown that there were minor fluctuations in the trajectory. With the proceeding of LHS batches, the number of samples covered increases gradually. Correspondingly, the cumulative performance indices converged to a stable value gradually. The average performance in the feasible region could exhibit convergence characteristics under the cumulative effect, and the variability in the performance of the methods is thus eliminated. This could reveal the relative performance between the two methods.

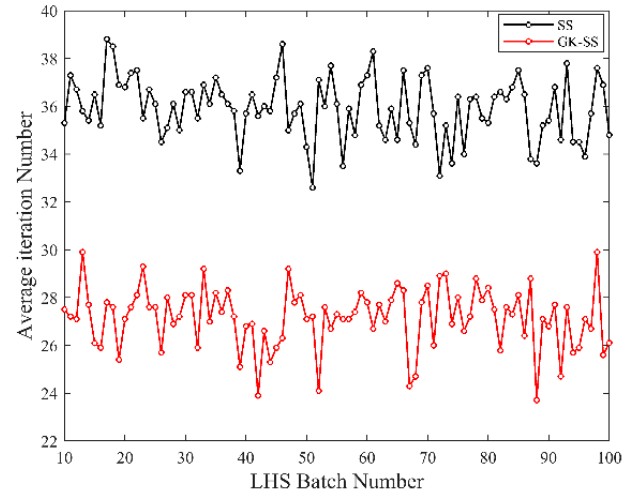

(a)

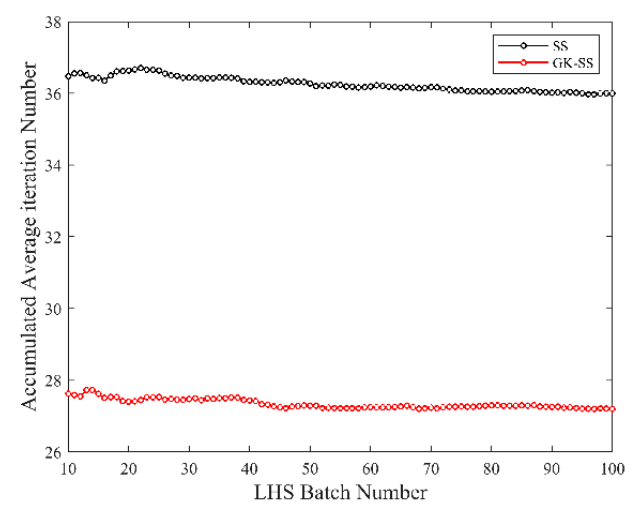

(b)

Figure 18. Average iteration number distribution in the test: (a) dot diagram of the average iteration numbers; (b) Accumulated averaged iteration number.

Although the GK-SS behaved better in the optimization efficiency, the optimization results obtained by it may not be improved. Figure 19a shows the average optimal ITAE index obtained of each batch for the SS and the GK-SS, and Figure 19b shows their accumulated average optimal ITAE index. The trajectories indicated that the GK-SS behaved a little worse than the SS on the final optimal outputs. However, the deficiency was so minor that it still can be concluded that the two methods could obtain similar optimal results. The deficiency phenomenon was reasonable, because the principle of the GK-SS lies in the efficiency promotion to enhance the economy index on the iteration number, not in the promotion of the optimal results. Considering that a little sacrifice of the optimality can be exchanged for the improvement of the economy index, GK-SS is still an efficient method. 


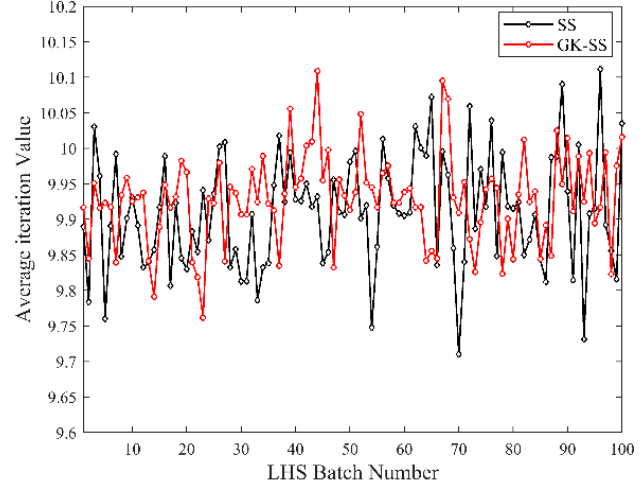

(a)

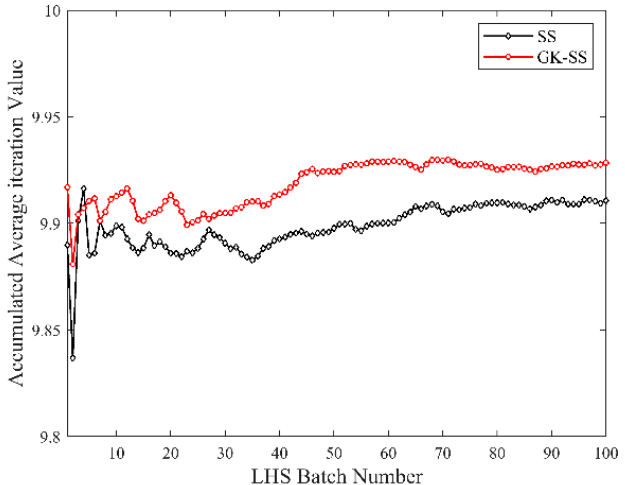

(b)

Figure 19. Average optimal ITAE index distribution in the test: (a) dot diagram of the average optimal ITAE index; (b) Accumulated averaged optimal ITAE index.

To sum up, the performance efficiency indices are demonstrated in Figure 20. Figure 20a illustrates the average iteration number of the two methods. Downward trends in iteration number statistics of the GK-SS relative to the traditional simplex search method suggested that the revised method is relatively more efficient. It can be observed that the average iteration number diminished significantly, with a reduction of $22.9 \%$.

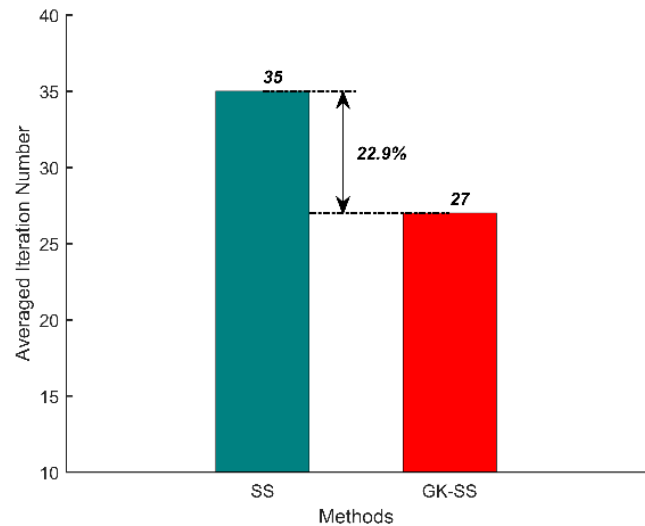

(a)

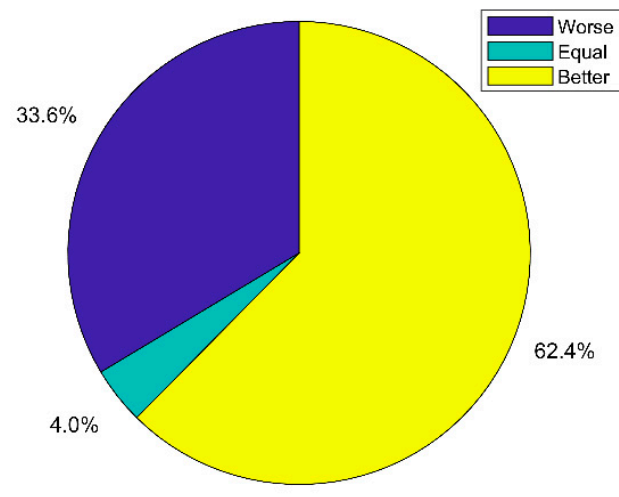

(b)

Figure 20. Performance evaluation of GK-SS vs. traditional simplex search method (SS) by statistics. (a) Averaged iteration number. (b) Superiority ratio by direct performance comparison.

Figure 20b demonstrates the performance statistics of the GK-SS relative to the traditional method. It can be seen that the GK-SS behaved better than the traditional method in almost $63 \%$ of cases. Accordingly, the results showed that the revised method had an appreciable effect on efficiency promotion. Hence, it was indicated that the GK-SS is efficient for the performance optimization of the steam generator level control.

\section{Conclusions}

In this study, a revised simplex search method, GK-SS, was proposed to optimize the performance of the steam generator level control system. With the data-driven optimization scheme that we proposed, the GK-SS deals with the act of data-driven optimization entirely. Hence, there are two critical modifications with the GK-SS-based data-driven method, covering all the dimensions on the efficiency promotion of the data-driven method. With the two modification mechanisms, the historical information generated in the optimization progress was fully utilized to guide the search direction and monitor the optimization progress. The simulation experiment indicated that the revised method could quickly converge to the optimal parameter settings. Further, the deliberately designed statistical 
experiments also showcased that the revised method significantly fortified the efficiency of the data-driven optimization method. Thus, it can be concluded that the revised simplex search method was effective and efficient in controller parameters optimization of the SG level control system. This method can be applied to other similar controller parameters optimization problems in the process control of all kinds of nuclear power plants.

Author Contributions: Conceptualization, X.K., C.S., H.L. and J.L.; data curation, X.K., P.G. and Y.F.; formal analysis, X.K.; funding acquisition, X.K., C.S., H.L. and J.L.; investigation, X.K., C.S. and P.G.; methodology, X.K. and C.S.; project administration, X.K., C.S., H.L. and J.L.; resources, X.K., C.S. and H.L.; software, P.G. and Y.F.; supervision, X.K., C.S. and H.L.; validation, X.K. and P.G.; visualization, X.K. and P.G.; writing—original draft, X.K., P.G. and Y.F.; writing-review and editing, X.K., C.S., H.L. and P.G. All authors have read and agreed to the published version of the manuscript.

Funding: This research was funded by the program of the State Key Laboratory of Nuclear Power Safety Monitoring Technology and Equipment of China (K-A2020.412), and the Natural Science Foundation of Fujian Province, grant numbers 2021J011205 and 2018J01564.

Institutional Review Board Statement: Not applicable.

Informed Consent Statement: Not applicable.

Data Availability Statement: Not applicable.

Conflicts of Interest: The authors declare no conflict of interest.

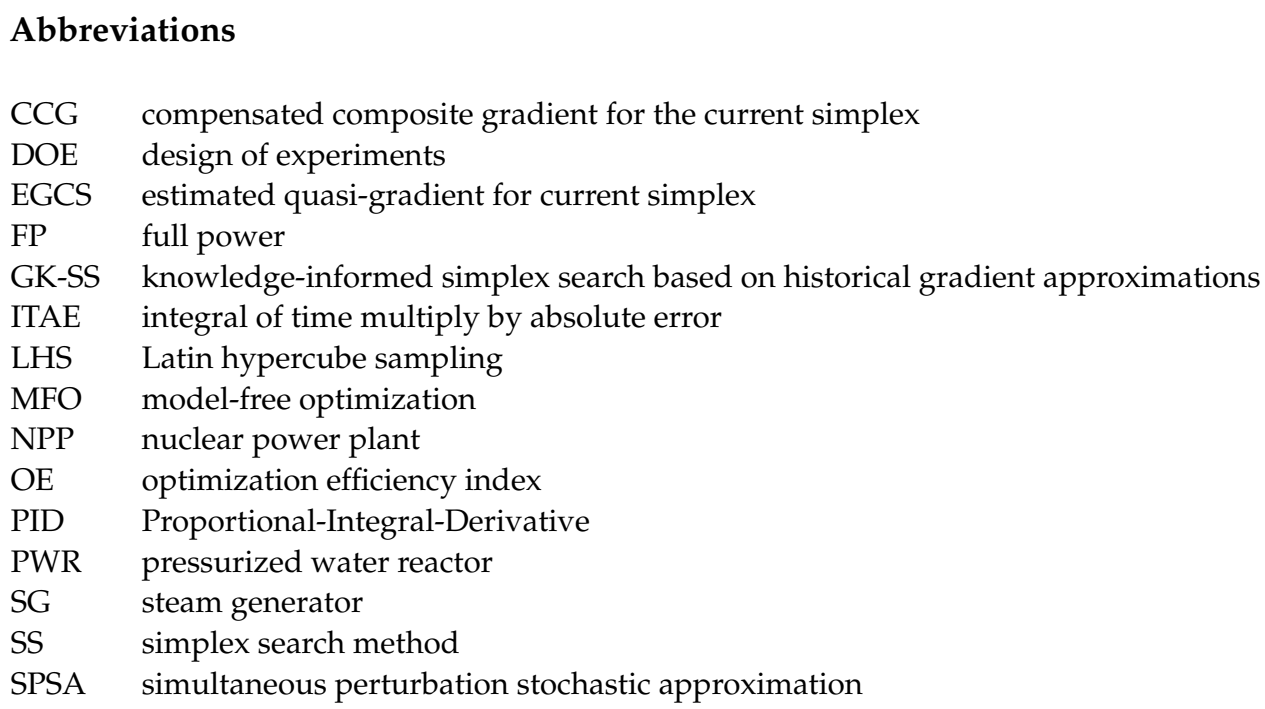

\section{References}

1. Wu, S.; Wang, P.; Wan, J.; Xinyu, W.; Zhao, F. Parameter Optimization for AP1000 Steam Generator Feedwater Control System Using Particle Swarm Optimization Algorithm. In Proceedings of the 24th International Conference on Nuclear Engineering, Charlotte, NC, USA, 26-30 June 2016. [CrossRef]

2. Irving, E.; Miossec, C.; Tassart, J. Towards Efficient Full Automatic Operation of the PWR Steam Generator with Water Level Adaptive Control. In Boiler Dynamics and Control in Nuclear Power Stations, Bournemouth; Thomas Telford Publishing: London, UK; pp. 309-329. [CrossRef]

3. Salehi, A.; Safarzadeh, O.; Kazemi, M.H. Fractional Order PID Control of Steam Generator Water Level for Nuclear Steam Supply Systems. Nucl. Eng. Des. 2019, 342, 45-59. [CrossRef]

4. Ablay, G. Robust Estimator-based Optimal Control Designs for U-Tube Steam Generators. Trans. Inst. Meas. Control. 2015, 37, 636-644. [CrossRef]

5. Xu, Y.; Chen, J.; Yu, H.; Wang, Z. Research on Feedforward Compensation for Steam Generator Level Control System Manual/Automatic Switch. Hedongli Gongcheng/Nucl. Power Eng. 2021, 42, 140-144. [CrossRef]

6. Hui, J.W.; Ling, J.; Dong, H.; Wang, G.X.; Yuan, J.Q. Distributed Parameter Modeling for the Steam Generator in the Nuclear Power Plant. Ann. Nucl. Energy. 2021, 152. [CrossRef]

7. Zhang, D.; Cai, W.; Xie, C.; Liu, P.; Liu, C. Research on Automatic Optimization Software for PID Parameters of Nuclear Power Digital Control System PID. Hedongli Gongcheng/Nucl. Power Eng. 2020, 41, 77-81. [CrossRef] 
8. Zhuang, D.P.A. Automatic Tuning of Optimum PID Controllers. IEE Proc. -D 1993, 140, 216-224. [CrossRef]

9. Wei, Z.J.; Chai, T. A Survey of Advanced PID Parameter Tuning Methods. Acta Autom. Sinica 2000, 26, $347-355$.

10. Åström, K.J.; Hägglund, T. The Fture of PID Control. Control Eng. Pract. 2001, 9, 1163-1175. [CrossRef]

11. Zheng, Y.; Zhang, Y.; You, K.; Zhao, M.; Li, Y.; Chen, G.; Yan, X. Research on Improved Auto-Tuning of a PID Controller Based on Phase Angle Margin PID. Hedongli Gongcheng/Nucl. Power Eng. 2020, 41, 108-113. [CrossRef]

12. Demerdash, N.A.; El-Hameed, M.A.; Eisawy, E.A.; El-Arini, M.M. Optimal Feed-Water Level Control for Steam Generator in Nuclear Power Plant based on Meta-Heuristic Optimization. J. Radiat. Res. Appl. Sci. 2020, 13, 468-484. [CrossRef]

13. Formentin, S.; Karimi, A. A Data-Driven Approach to Mixed-Sensitivity Control with Application to An Active Suspension System. IEEE Trans. Ind. Inform. 2012, 9, 2293-2300. [CrossRef]

14. Procházka, H.; Gevers, M.; Anderson, B.D.; Ferrera, C. Iterative Feedback Tuning for Robust Controller Design and Optimization. In Proceedings of the 44th IEEE Conference on Decision and Control, Seville, Spain, 15 December 2005. [CrossRef]

15. Sala, A.J.A. Integrating Virtual Reference Feedback Tuning into A Unified Closed-Loop Identification Framework. Automatica. 2007, 43, 178-183. [CrossRef]

16. Lei, T.; Hou, Z.; Ren, Y. Data-Driven Model Free Adaptive Perimeter Control for Multi-Region Urban Traffic Networks With Route Choice. IEEE Trans. Intell. Transp. Syst. 2019, 1-12. [CrossRef]

17. Hou, Z.; Zhu, Y.I. Controller-dynamic-linearization-based Model Free Adaptive Control for Discrete-Time Nonlinear Systems IEEE Trans. Ind. Inform. 2013, 9, 2301-2309. [CrossRef]

18. Hou, Z.-S.; Wang, Z. From Model-Based Control to Data-Driven Control: Survey, Classification and Perspective. Inf. Sci. 2013, 235, 3-35. [CrossRef]

19. Gao, Z.; Saxen, H.; Gao, C. Guest editorial: Special section on data-driven approaches for complex industrial systems. IEEE Trans. Ind. Inform. 2013, 9, 2210-2212. [CrossRef]

20. Gao, Z.; Chen, M.Z.; Zhang, D.J.P. Special Issue on “Advances in Condition Monitoring, Optimization and Control for Complex Industrial Processes". Processes 2021, 9, 664. [CrossRef]

21. Gao, Z.; Nguang, S.K.; Kong, D.-X.J.C. Advances in Modelling, Monitoring, and Control for Complex Industrial Systems Complexity 2019, 2019, 2975083. [CrossRef]

22. Gao, Z.; Kong, D.; Gao, C. Modeling and Control of Complex Dynamic Systems: Applied Mathematical Aspects. J. Appl. Math. 2012, 2012, 869792. [CrossRef]

23. Kong, X.; Xiao, Y.; Qian, L.; Su, L.; Chen, B.; Xu, M. Performance Optimization for Steam Generator Level Control Based on A Revised Simultaneous Perturbation Stochastic Approximation Algorithm. In Proceedings of the 2018 3rd International Conference on Intelligent Green Building and Smart Grid (IGBSG), Yilan, China, 22-25 April 2018. [CrossRef]

24. Kong, X.-S.; Guo, J.-M.; Zheng, D.-B.; Yang, J.-F.; Zhang, J.; Fu, W. An Improved-SPSA Quality Control Method for Medium Voltage Insulator SPSA. Gao Xiao Hua Xue Gong Cheng Xue Bao/J. Chem. Eng. Chin. Univ. 2020, 34, 1500-1510. [CrossRef]

25. Kong, X.; Guo, J.; Zheng, D.; Zhang, J.; Fu, W. Quality Control for Medium Voltage Insulator via a Knowledge-Informed SPSA Based on Historical Gradient Approximations. Processes 2020, 8, 146. [CrossRef]

26. Kong, X.; Ma, Z. A Novel Method for Controllers Parameters Optimization of Steam Generator Level Control. In Proceedings of the 21st International Conference on Nuclear Engineering, Chengdu, China, 29 July-2 August 2013. [CrossRef]

27. Kong, X.; Zheng, D. A Knowledge-Informed Simplex Search Method Based on Historical Quasi-Gradient Estimations and Its Application on Quality Control of Medium Voltage Insulators. Processes 2021, 9, 770. [CrossRef]

28. Dai, X.; Gao, Z. From model, signal to knowledge: A Data-Driven Perspective of Fault Detection and Diagnosis. IEEE Trans. Ind. Inform. 2013, 9, 2226-2238. [CrossRef]

29. Zhang, D.; Gao, Z. Improvement of Refrigeration Efficiency by Combining Reinforcement Learning with A Coarse Model. Processes 2019, 7, 967. [CrossRef]

30. Nelder, J.A.; Mead, R. A Simplex-Method for Function Minimization. Comput. J. 1965, 7, 308-313. [CrossRef]

31. Tan, W. Water Level Control for A Nuclear Steam Generator. Nucl. Eng. Des. 2011, 241, 1873-1880. [CrossRef] 